\title{
El entrenamiento de profesionales de atención primaria en consejería de hábitos de vida saludable no redujo el reporte de cambio por parte de los pacientes
}

Training primary care professionals in counseling for healthy lifestyles adoption did not reduced patients reporting change

Butler C y col. BMJ 2013;346:f1191

\section{Objetivos}

Evaluar el efecto de entrenar a profesionales de atención primaria en el consejo de cambios de conducta en pacientes que refieren algunas de las siguientes conductas de riesgo: tabaquismo, consumo de alcohol, ausencia de actividad física y alimentación inadecuada. ${ }^{1-5}$

\section{Diseño, lugar y pacientes}

Ensayo aleatorizado por clusters, utilizando a las clínicas generales de Gales como unidad de aleatorización.

Se incluyeron 53 médicos generales y 27 enfermeras de clínicas generales (uno en cada clínica excepto en una), y 1.827 pacientes con al menos una conducta de riesgo.

\section{Intervención}

Se realizó consejería sobre el cambio de conducta a través de entrevistas motivacionales a pacientes. Los médicos fueron entrenados previamente, utilizando un programa de aprendizaje mixto llamado Talking Lifestyles.

\section{Medición de resultados principales}

Se determinó la proporción de pacientes que informaron realizar cambios beneficiosos en al menos una de las cuatro conductas de riesgo dentro de los tres meses de seguimiento.

\section{Resultados principales}

Se incluyeron 1.308 pacientes de 13 clínicas del grupo intervención y 1.496 de 14 clínicas de la rama control, de los cuales el $76 \%$ y $72 \%$ respectivamente aceptaron participar. De ellos $831(84 \%)$ y $996(92 \%)$ respectivamente presentaron al menos una conducta de riesgo.

En relación al resultado primario, no se observaron diferencias significativas a los tres meses de seguimiento ni tampoco en los parametros bioquímicos y biométricos analizados a los 12 meses. Ver tabla 1.

Los pacientes que participaron de una consulta con un médico entrenado refirieron haber discutido conductas saludables con el profesional y haber tenido una intención de cambio mayor OR 12,44 (IC95\% 5,85 a 26,46) y OR 2,88 (IC95\% 2,05 a 4,05).

Tabla 1: resultados principales de cambio de comportamiento en al menos una conducta de riesgo a los tres y 12 meses de seguimiento.

\begin{tabular}{|c|c|c|c|c|c|c|}
\hline \multirow{2}{*}{$\begin{array}{l}\text { Cambios en el } \\
\text { comportamiento }\end{array}$} & \multicolumn{3}{|c|}{ Resultados a los tres meses de seguimiento } & \multicolumn{3}{|c|}{ Resultados a los 12 meses de seguimiento } \\
\hline & $\begin{array}{l}\text { Grupo control } \\
\mathrm{N}(\%)\end{array}$ & $\begin{array}{l}\text { Grupo intervención } \\
\mathbf{N}(\%)\end{array}$ & $\begin{array}{l}\text { OR } \\
\text { (IC 95\%) }\end{array}$ & $\begin{array}{l}\text { Grupo control } \\
\text { N (\%) }\end{array}$ & $\begin{array}{l}\text { Grupo intervención } \\
\mathrm{N}(\%)\end{array}$ & $\begin{array}{l}\text { OR } \\
\text { (IC 95\%) }\end{array}$ \\
\hline $\begin{array}{l}\text { Pacientes que realizaron al } \\
\text { menos un cambio }\end{array}$ & $592(59,4)$ & $469(56,4)$ & \multirow[t]{2}{*}{$1,12(0,9$ a 1,39$)$} & $600(60,2)$ & $495(59,4)$ & \multirow[t]{2}{*}{$1,03(0,83$ a 1,28$)$} \\
\hline $\begin{array}{l}\text { Pacientes que no } \\
\text { realizaron cambios }\end{array}$ & $404(40,6)$ & $362(43,6)$ & & $396(39,8)$ & $337(40,6)$ & \\
\hline
\end{tabular}

\section{Conclusiones}

El entrenamiento de médicos de atención primaria a través de un breve programa de enseñanza no aumentó el reporte de cambios saludables por parte de los pacientes a los tres meses, ni modificó la biometría y bioquímica a los 12 meses. Sí

se detectó un aumento en la percepción de discusión de estos temas en la consulta, con un incremento en la intención de cambio y en la cantidad de intentos realizados.

Fuente de financiamiento: National Prevention Research Initiative con el apoyo de British Heart Foundation; Cancer Research UK.

\section{Comentario}

Teniendo en cuenta que el $80 \%$ de la población del Reino Unido consulta anualmente a clínicas generales y en el contexto de las recomendaciones internacionales de "hacer valer cada consulta", se buscó evaluar la efectividad de médicos entrenados en dar consejo oportuno sobre conductas saludables. Sólo un pequeño porcentaje (12\%) de las clínicas en la rama intervención completó todas las etapas de entrenamiento. Esto podría haber diluido el efecto de la intervención, pero por otro lado fortalece la validez externa del estudio.

A la hora de evaluar los resultados, y observando que no hubieron diferencias entre los grupos, no se puede desestimar el efecto que pudo haber tenido el rastreo en los pacientes, ya que podría haber funcionado como una cointervención.

Si evaluamos el objetivo primario, que era el reporte de cambios beneficiosos a los 3 meses, el seguimiento hasta este punto fue del $80,4 \%$ para el grupo intervención y del $80,5 \%$ para la rama control; pero el seguimiento completo a los 12 meses cayó significativamente siendo de $51,1 \%$ y $54,6 \%$ respectivamente; la ausencia de respuesta podría considerarse como una negativa a los cambios de conducta.

Si bien este estudio no pudo demostrar cambios duraderos, se debe tener en cuenta que la intervención se realizó en una única consulta de rutina sin otra intervención adicional, lo cual indicaría que esto no sería suficiente, pero no por ello debería dejar de realizarse.

Queda pendiente en próximos estudios determinar si la presencia de intervenciones adicionales, mayor entrenamiento, seguimiento más estructurado u otras conductas podrían demostrar cambios a largo plazo.

María Teste [ Salud Comunitaria Trenque Lauquen. mariteste@gmail.com ]

Teste M. El entrenamiento de profesionales de atención primaria en consejería de hábitos de vida saludable no redujo el reporte de cambio por parte de los pacientes. Evid Actual Pract Ambul. 2015;18(3):73. Jul-Sep. Comentado de: Butler C, y col. Training practitioners to deliver opportunistic multiple behaviour change counselling in primary care: a cluster randomised trial. BMJ. 2013 Mar 19;346:f1191. PMID:23512758.

\section{Referencias}

1. Roe L, y col. Dietary intervention in primary care: validity of the DINE method for diet assessment. Fam Pract 1994;11:375-81.

2. Cappuccio FP, y col. Estimation of fruit and vegetable intake using a two-item dietary questionnaire: a potential tool for primary health care workers. Nutr Metab Cardiovasc Dis 2003;13:12-9.

3. Craig CL, y col. International physical activity questionnaire: 12-country reliability and validity. Med Sci Sports Exerc 2003;35:1381-95.

4. Kozlowski LT, y col. Predicting smoking cessation with self-reported measures of nicotine dependence: FTQ, FTND, and HSI. Drug Alcohol Depend 1994;34:211-6.

5. Bush K, y col The AUDIT alcohol consumption questions (AUDIT-C): an effective brief screening test for problem drinking. Ambulatory Care Quality Improvement Project (ACQUIP). Alcohol Use Disorders Identification Test. Arch Intern Med 1998;158:1789-95. 